\title{
The Chaotic Universe's Cosmogony: the Universe's Perpetuum Mobile, Multivariance of the Universe and the "Boiling" Universe Hypothesis
}

\author{
Alexander V. Sosnitsky ${ }^{1}$, Anatoly I. Shevchenko \\ ${ }^{1}$ Department of Computer Technologies, Berdyansk State Pedagogical University,4, Schmidt street, 71110, Berdyansk, Ukraine \\ ${ }^{2}$ Institute of AI Problems of the MES and NAS of Ukraine, 11/54, Mala Zhitomirska street, 01001, Kiev, Ukraine
}

\begin{abstract}
How to cite this paper: Sosnitsky, A.V. \& Shevchenko, A.I. (2018) The Chaotic Universe's Cosmogony: the Universe's Perpetuum Mobile, Multivariance of the Universe and the "Boiling" Universe Hypothesis. Journal of Applied Mathematics and Computation, 2(3), 84-92. http://dx.doi.org/10.26855/ja mc.2018.03.002

Corresponding author: A lexander V. Sosnitsky, Department of Computer Technologies, Berdyansk State Pedagogical University,4, Schmidt street, 71110, Berdyansk, Ukraine

E-mail: sosnitsky.ukr@yandex.ua
\end{abstract}

\begin{abstract}
The paper investigates the complex universalization of the Universe's cosmogony, covering all stages of existence of the Universe and resolving the main external and internal contradictions unlike particular axiomatic theories. The structural formalism of cosmogony is obtained, developing generally accepted theories by means of fundamentally important concepts of Absolute Chaos, Harmon, Universe's Perpetuum Mobile, multivariance of the Universe, hypotheses of the "boiling" Uni verse, inverted Universe's cosmogony, etc. A great number of degrees of infinity and the possibility of unlimited multivariance of the Universe as well as the other interpretation of the known scientific data are substantiated. The Universe's cosmogony has great general scientific importance and can be used in different fields of knowledge.
\end{abstract}

Keywords

Universe's Model, Universe's Cosmogony, Chaos, Harmony, Perpetuum Mobile.

\section{Introduction}

Universalization of knowledge develops axiomatic (in more general terms - dogmatic) system of particular knowledge by the transition to the unified comprehensive system of concepts, naturally opening up new possibilities and enriching the earlier acquired knowledge by the new highest universal concepts with more common and usually opposite radical consequences [1].

The universal paradigm of knowledge as a new scientific school only begins its formalization. However, even right now the initial universal meta-concept allows substantiating many important conclusions inaccessible by the other methods. At the same time, more general identifiable Universe's concepts have a larger area of action and fundamental consequences [2].

It is the purpose of this paper to investigate the universal cosmogony of the Universe following from the uniform universal formalism - the Universal (more exact - Universe's) Theory (Model) obtained according to the new universal scientific paradigm.

Today there are a great number of various partial dogmatic cosmological theories. However, all of them have severe shortcomings: they are poorly coordinated among themselves, come from challenging research, are to a great extent of a hypothetical character, contain many internal contradictions and have small conceptual and factual basis of research.

The main one is the hypothesis about the Big Bang and inflation of the Universe from a certain singular point which is allegedly confirmed by the increase in the red shift of spectral lines of stars as they move away from us [3-6]. However, the hypothesis faces insoluble problems of an explanation of the initial beginning, borders and termination of the Universe and, it is subjective relative to an observer.

The theories of "inflation/swelling" of the Universe evenly all over the space are more substantiated [7], however, they do not also explain the important properties of a cosmology mentioned above. The standard [3-7] and string [8] theories 
explain the origin of the Universe at the microcosm level, but they use already existing conceptual and methodological properties [9].

The Universal Theory differs fundamentally from them and describes consistently the origin and evolution of the Universe from Absolute Chaos and coordinates perfectly well with the main factual data and other Universe's hypotheses. It uses elementary notions of an entity and relation (conceptually defined in [1]), ensuring homogeneous formalization of any complexity by means of the modified ER diagrams (the arrows of which correspond to the copies of entities) which are naturally transferred to the set descriptions allowing an unambiguous complete and precise formalization.

\section{The Universe's Axiom and the Universe's Cosmogony}

Universalization is based on many important principles of the structure of the Universe and its cognition, the initial of which is an antique representation of the Universe as a single whole consisting of the invisible abstract strictly formal World (AW) of categories (abstracts, concepts) in the form of the Universe's abstract "pyramid" (the UAP) which are built in the visible chaotic real World (RW) of phenomena (Fig. 1).

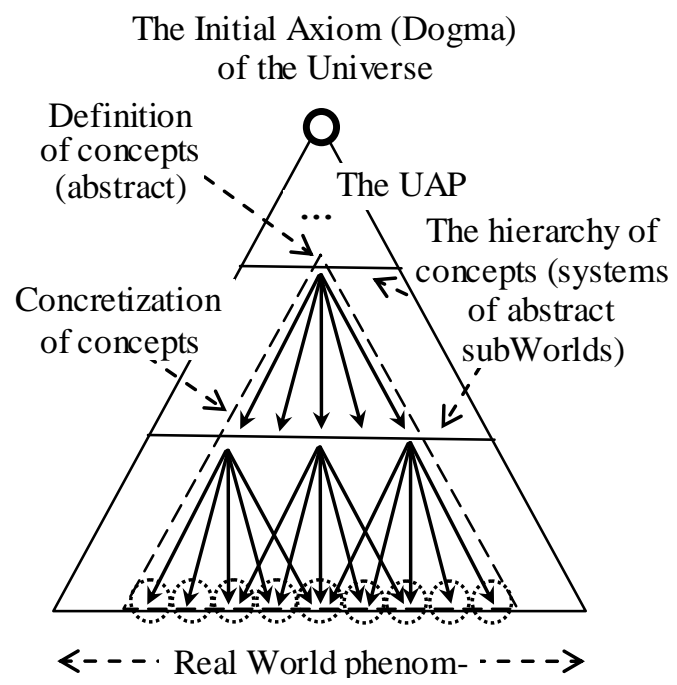

Figure 1. The scheme of the Universe's abstractness and science universalization

Hypothetically, the AW and, therefore, the Universe come from the uniform initial universal concept - the Universe's Axiom (the Universe's Dogma) generating the system of Universe's concepts of the UAP. The Universe's Axiom is supposed in the form of the Harmon that is self-defined as an infinitely recursively enclosed complete (C) infinite (I) directed (D) graph (G) CIDG (CIDG), the vertices of which are the same graphs (Fig. 2).

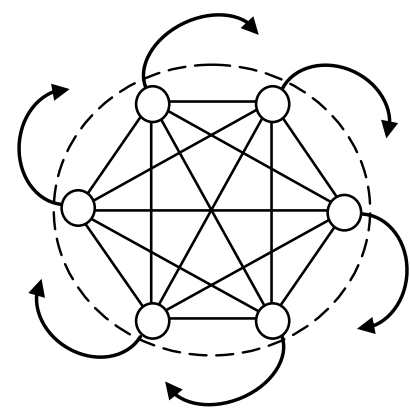

Figure 2. The scheme of the Harmon (for $n=6$, the number of internal entities is infinite). The arrows symbolize the equivalence of the Harmon's internal entities to the Harmon itself 
The Harmon (Absolute Everything) comes from Absolute Chaos (Absolute Nothing), which can transform into each other (condensation / decondensation) owing to an indistinguishability of the Harmon and its components, similar to physical statistics particles with integer spin (bosons).

According to universal definitions harmony is understood as the presence of relations, and the chaos - as the absence of relations.

Absolute Chaos is absolutely symmetric, but under some conditions it decondenses into the Harmon which possesses initial internal asymmetry (the hierarchy of enclosure) and, therefore, is unstable and then disintegrates into Quasi-Chaos (the Universe) with some residual Harmony. At the same time the UAP is formed and there arise Space, Time and Matter (STM-Complex) (Fig. 3) which harmonize the Universe back into the Harmon that condenses into Absolute Chaos then (Fig. 4).

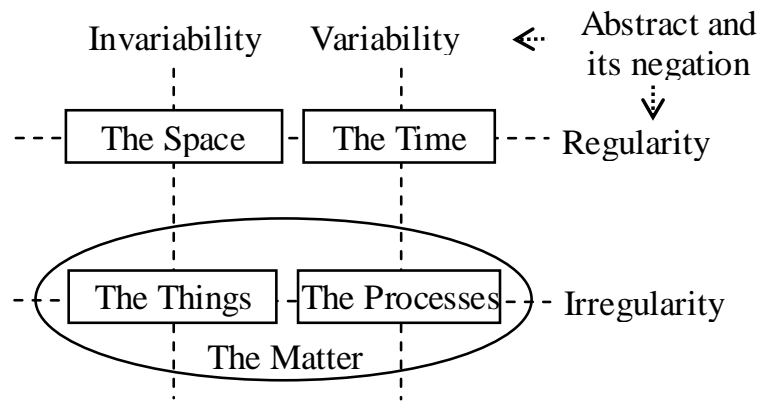

Figure 3. Classification of Complexes of the real World depending on the abstracts and their negations - variability / invariability and regularity / irregularity

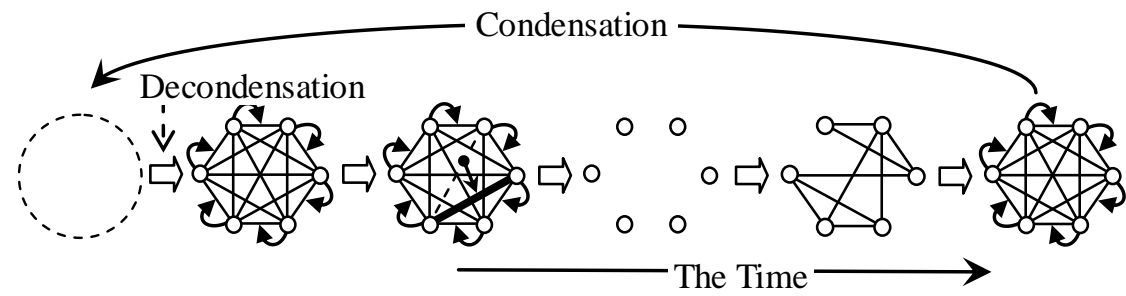

The Abso- The Start of internal The Qua- The Self- harmo- The lute Chaos Harmon decondensation si-Chaos nizing Universe Harmon

Figure 4. The scheme of the Universe's cosmogony

As the carrier capacity of the mechanism of decondensation is limited in time, the Universe must begin from the zero state of Space, Time and Matter corresponding to the point of initial singularity (decondensation of Absolute Chaos in the Harmon) of the Universe.

However, if we consider that the Universe is conceptually homogeneous with the uniform and invariable UAP, then it increases solely at the expense of the RW, represented by the general STM-Complex that is divided into the specified three particular Complexes of Space, Time and Matter. As far as the Universe is self-defined and, by definition, does not contain external entities but exclusively internal ones, then every portion of harmony from the Harmon disintegrates into corresponding mutually proportional portions of Space, Time and Matter that are thus connected among themselves and subjective for the Universe. It means that each produced portion of Time corresponds to the singled out portions of Space and Matter with some restricted normalized productivity in Time (most probably compared with the speed of light). Time, Space and Matter are highly subjective for the Universe and do not go beyond its limits where there is nothing for it.

Thus, 1) carrier capacity of the mechanism of decondensation is really limited 2) by a certain normalized quantity, and 3) the points of singularity in the Universe exist and 4) they are accompanied by the release of Space, Time and Matter, 5) with the help of which they are indirectly observed. 
However, judging by the Harmon structure, the quantity of singularity points must be infinite. At the initial moment it must divide instantly in Space and form a unified common infinite boundless Space that is saturated with singularity points continuously and at a constant normalized productivity in Time.

According to this model, the Universe is divided into the expanding zones of the continuous action of singularity points with the approximately identical mutually proportional density of Space, Time and Matter which, under their own laws, are redistributed in the Universe then.

Further evolution of these zones is associated with the emergence and development of life (intelligence, 3rd Class of phenomena, according to Tab. 1) that, by means of active harmonization, sequentially reaches the state of the Harmon in zones and completes the cycle of its existence described below. Herewith, the corresponding point of singularity will act and allocate the resources of Space, Time and Matter just as many as it is required to complete that cycle.

The cosmogony in question is largely of a hypothetical character, but it does not contain fundamental contradictions of the other (especially extreme) theories and is well confirmed by both the Universal Theory and real data.

The observable Space has a complex quasi-regular arrangement of galaxies that obey the laws of ultraheavy elementary particles with the deviation from the properties of the real observable Matter in favor of certain hidden (dark) Matter, in the centers of galaxies there are generators of large masses of Matter (and, therefore, Space and Time) that spread all over the Universe and feed its development. Matter is directly observed in generating by quasars, Time - indirectly in the movement of Matter, Space - indirectly in the continuously accelerating with the distance omnipresent "swelling" that increases mutual recession of Matter and, as a consequence, the Doppler effect, to which energy expenditure (that has not been found yet) on movement in Space can be added.

Owing to the division of the general STM-Complex, the particular Complexes of Space, Time and Matter can be unevenly distributed all over the Universe and cause effects, similar to the relativistic Lorentz transformation.

The UAP is an instrument for achieving by the RW the state of the Harmon and, in the first approximation, supposed to be invariable throughout the whole Time. The origin of the UAP is not investigated in this paper.

The physics of the specified properties and mechanisms of action has been unknown so far, the exact relationship of the concepts has not been revealed, and part of them goes beyond our Universe and, perhaps, will never be understood. This cosmology remains hypothetical as well as any knowledge in general, but in such a representation the apparent external and internal contradictions are resolved and the distance among the concepts used is reduced. The contradictions seem to be surmountable (until the contrary is proved) while offering further more precise definition of the conceptual and factual basis of the research.

\section{The Universe's Perpetuum Mobile and Multivariance of the Universe}

According to the classical definition, the pair of the Harmon / Absolute Chaos and the mechanism of a decondensation are the Universe's Perpetuum Mobile as an inexhaustible source of the whole entity in the Universe from nothing, which is impossible in a particular case (an entropic and energetic one, Classes 1-2 in Table 1) and possible in the universal sense (Classes 3 and above in Table 1).

The existence of an inexhaustible source of the entity changes radically cosmogony. The possibility of precise preservation of the Past that differs from the Present only by the absence of Time is an important consequence. Any point of the Past can obtain the other development in the Future by means of switching on Time in the new circumstances, thus obtaining unlimited multivariational existence of the Universe from beginning to end, from which it is possible to choose the best for continuation as per a certain criterion.

Real access to the whole Universe throughout the whole Time is vitally important for the Class 4 phenomena and opens up a possibility to reach the state of the Harmon according to the universal classification of phenomena in Time (Table $1)$. 
Table 1. The uni versal harmonic classification of phenomena in Time

\begin{tabular}{|c|c|c|c|c|c|}
\hline Class & Name & $\begin{array}{c}\text { Internal } \\
\text { structure }\end{array}$ & Harmonious resource & $\begin{array}{c}\text { Harmony } \\
\text { type }\end{array}$ & $\begin{array}{c}\text { Characteristic } \\
\text { quantity }\end{array}$ \\
\hline 1 & $\begin{array}{c}\text { Quasi-Chaos } \\
\text { (Thermodynamics) }\end{array}$ & No & $\begin{array}{c}\text { Real relations in the Pre- } \\
\text { sent }\end{array}$ & Starting & Entropy \\
\hline 2 & $\begin{array}{c}\text { Natural selection } \\
\text { (Mechanics) }\end{array}$ & Present & $\begin{array}{c}\text { + virtual relations during } \\
\text { the interval in the Past }\end{array}$ & Passive & Energy \\
\hline 3 & Life (Intelligence) & $\begin{array}{c}\text { Copy of } \\
\text { STM-Complex }\end{array}$ & $\begin{array}{c}\text { + virtual relations during } \\
\text { the interval in the Future }\end{array}$ & Active & Harmony \\
\hline 4 & $\begin{array}{c}\text { The highest } \\
\text { Intelligence }\end{array}$ & $\begin{array}{c}\text { A real } \\
\text { STM-Complex }\end{array}$ & $\begin{array}{c}\text { + real Present on all } \\
\text { Time axis }\end{array}$ & Highest & Harmony \\
\hline 5 & Harmon & Absolute & Absolute connectedness & Absolute & Harmony \\
\hline 6 & Absolute Chaos & No & There are no relations & No & No \\
\hline
\end{tabular}

\section{Universal Harmonic Time Classification of Phenomena}

The physical structure of the UAP has remained a fundamental scientific problem since Aristotle. It escaped the attention of modern science and requires special research that can possibly be carried out by way of universalization as the closest similarity to the Universe.

The UAP is an exclusive instrument of existence and harmonization of the Universe according to the superlaw of general harmony (connectivity) that is observed everywhere in nature. This superlaw is directed towards establishing every possible connectivity of all the phenomena up to the achievement of the Universe's purpose - absolute harmony of the Harmon.

Phenomena exist in the Complexes of Space, Time and Matter and have the infinite hierarchically enclosed structure of relations that is divided into three groups: 1) internal relations, 2) external relations and 3) relations among them (Fig. 5).

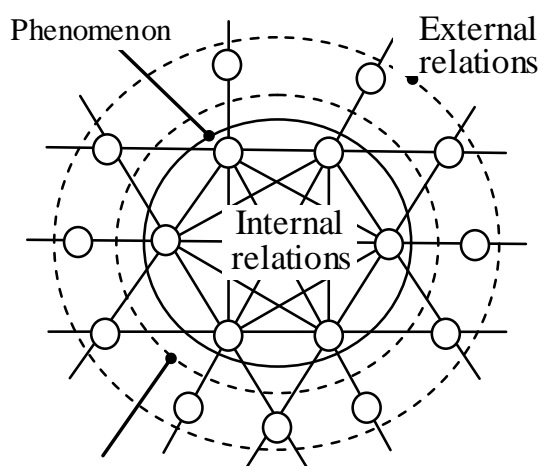

Relations between internal and external relations

Figure 5. The scheme of division of phenomena into three groups: internal relations, external relations, and relations among them

The increase in the latter group involves corresponding complication of internal relations that have the following distinct states that change radically the properties of phenomena (Table 1 ).

Class 1 does not activate the internal structure of phenomena by external relations and maintains relations only in the curre nt Present. Class 2 partially activates the internal structure of phenomena and in addition to Clas s 1 displays relations with the Past which create such a structure. Class 3 develops the internal structure of phenomena up to the level of subjective analog of STM-Complex and in addition to Class 2 displays relations with the Future by means of prediction. 
The Classes have characteristic quantities, defining interaction with Chaos and the Harmony (Table 2). Chaos of the enclosed phenomena in Class 1 grows, in Class 2 - conserves, in Class 3 - decreases in Time, and Harmony as an antipode of Chaos changes in the opposite direction, respectively. Thus, Chaos and Harmony mutually turn into each other, and Classes have different mechanisms of such transformation but in a manner that common Harmony of the bound phenomena tends towards the increase with the decrease of Chaos.

In fact, Class 1 is a converter of Harmony into Chaos, Class 3, on the contrary, - of Chaos into Harmony, and Class 2 maintains the balance between Chaos and Harmony [10].

Table 2. The characteristic quantities of harmonic Classes of phenomena in Time

\begin{tabular}{|c|c|c|c|}
\hline $\begin{array}{c}\text { Class of } \\
\text { phenomena }\end{array}$ & Name & $\begin{array}{c}\text { Characteristic quan- } \\
\text { tity }\end{array}$ & $\begin{array}{c}\text { Quality of phenomena } \\
\text { harmonization }\end{array}$ \\
\hline 1 & Thermodynamics & Entropy & Degradation \\
\hline 2 & Mechanics & Energy & Conservation \\
\hline 3 & Intelligence & Harmony & Development \\
\hline
\end{tabular}

\section{The Role of Phenome na Classes in the Universe's Cosmogony}

Fig. 6 shows the scheme of cosmogony of an action zone of one singular point. It can be assumed that such a point exists and generates a corresponding STM-Complex up to the emergence of the first 3 Classes of phenomena by a current moment of Time.

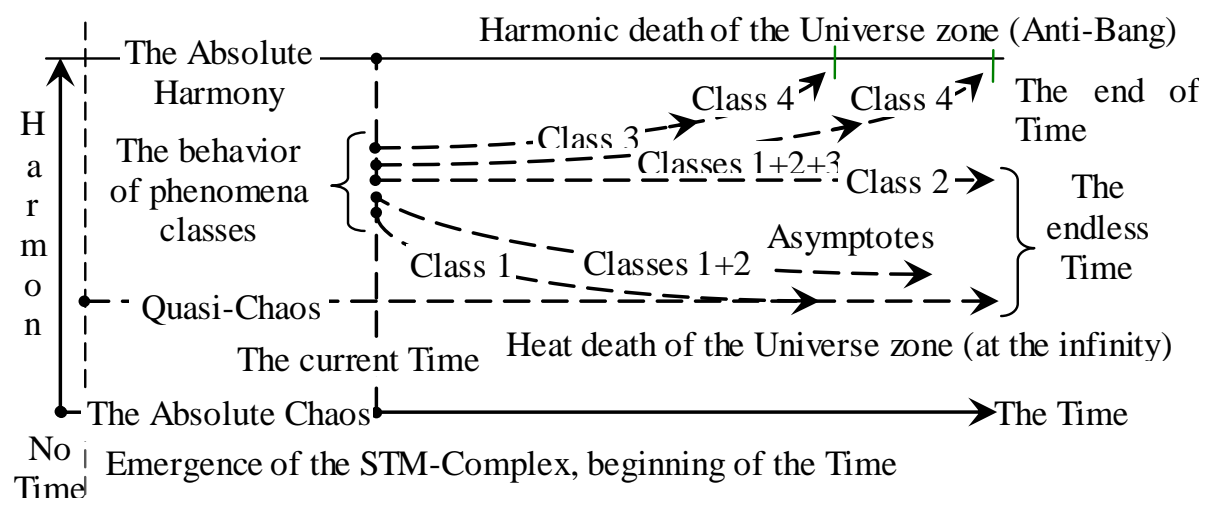

Figure 6. The scheme of development of an action zone of a singular point of the Universe

The phenomena develop further according to the following 5 schemes of development of mono- and mixed Classes of phenomena (Fig. 6) on account of the inheritance of properties of lower Classes by higher Classes (Tab. 1), the results of which are summarized in Table 3:

- Class 1 - the increase in Chaos due to Harmony decrease; result: phenomena degradation and heat death of the Universe in the asymptote.

- Class 2 - preservation of Chaos and Harmony; result: preservation of phenomena.

- Classes $1+2$ - combination of properties of Classes 1-2; result: degradation of phenomena and slow heat death of the Universe in the asymptote.

- Class 3 - the increase in Harmony due to the decrease of Chaos; result: that development of phenomena to the state of the Harmon and harmonic death of the Universe.

- Classes $1+2+3$ - combination of properties of Classes 1-3; result: slow development of phenomena to the Harmon's state and harmonic death of the Universe.

Class 1 is produced by a singular point and mechanically turns into Class 2 in an action zone of this point. By means of natural selection (passive harmonization) Class 2 develops into Class 3 of active harmonization and perfects itself up to the complete exhaustion of possibilities of cognition of the Universe with the virtual access to Time axis. The latter is not 
enough for transformation of the STM-Complex and the UAP into the Harmon. For this purpose direct access must be provided to them [11]. The superlaw of harmony generates the crisis of development of Class 3 into Class 4 . The latter gains such an access and uses it for the achievement of the Harmon which destroys the zone by means of condensation into Absolute Chaos on condition that the harmonizing ability of Class 4 exceeds the deharmonizing action of the singular point. Further speculation at this level is too variational and is not investigated in this paper.

Presumably, taking into account the increasing harmonizing ability of Classes, such transformations will occur with the acceleration up to the Anti-Bang at a final stage. The physics and mechanisms of such actions must become clearer while approaching the Harmon.

Table 3. The influence of phenomena Classes on the development of an action zone of a singular point in the Universe

\begin{tabular}{|c|c|c|c|c|}
\hline $\begin{array}{c}\text { Classes of } \\
\text { phenomena }\end{array}$ & Name & $\begin{array}{c}\text { Characteris- } \\
\text { tic quantities }\end{array}$ & $\begin{array}{c}\text { Results for phe- } \\
\text { nomena }\end{array}$ & $\begin{array}{c}\text { Results for the Uni- } \\
\text { verse zone }\end{array}$ \\
\hline 1 & Thermodynamics & Entropy & Disharmonization & Heat death \\
\hline 2 & Mechanics & Energy & Conservation & Stagnation \\
\hline $1+2$ & $\begin{array}{c}\text { Thermodynamics + } \\
\text { Mechanics }\end{array}$ & $\begin{array}{c}\text { Entropy }+ \\
\text { Energy }\end{array}$ & $\begin{array}{c}\text { Slow dishar- } \\
\text { monization }\end{array}$ & Slow heat death \\
\hline 3 & Intelligence & Harmony & $\begin{array}{c}\text { Limited har- } \\
\text { monization }\end{array}$ & $\begin{array}{c}\text { The development } \\
\text { problem of Class 3 } \\
\text { into Class 4 }\end{array}$ \\
\hline $1+2+3$ & $\begin{array}{c}\text { Thermodynamics + } \\
\text { Mechanics + Intel- } \\
\text { ligence }\end{array}$ & $\begin{array}{c}\text { Entropy + } \\
\text { Energy + } \\
\text { Harmony }\end{array}$ & $\begin{array}{c}\text { Slow limited } \\
\text { harmonization }\end{array}$ & $\begin{array}{c}\text { The development } \\
\text { problem of Class 3 } \\
\text { into Class 4 }\end{array}$ \\
\hline
\end{tabular}

\section{The Hypothesis of the "Boiling" Universe}

There seems to be most probable the existence of an infinite number of singular points and global division of the Universe into the corresponding zones of the specified cosmogony. The zones are continuously arising and disappearing in the Universe according to the scheme above similar to a boiling liquid. (Fig. 7)

As a result, the Universe is finite in Time for singular points, but in general it 1) is infinite in Time, 2) as well as in Space and Matter, 3) has always been and will be in Time, 4) has an infinite number of internal relatively independent divisions, 5) in each of which multivariance of trajectories of existence is possible 6) up to self-determination of its properties (similar to the Harmon) on condition that the properties on borders of zones are coordinated.

Penetration to that ultrahigh level of abstraction will open a lot of new knowledge, changing radically every time our notion of the Universe.

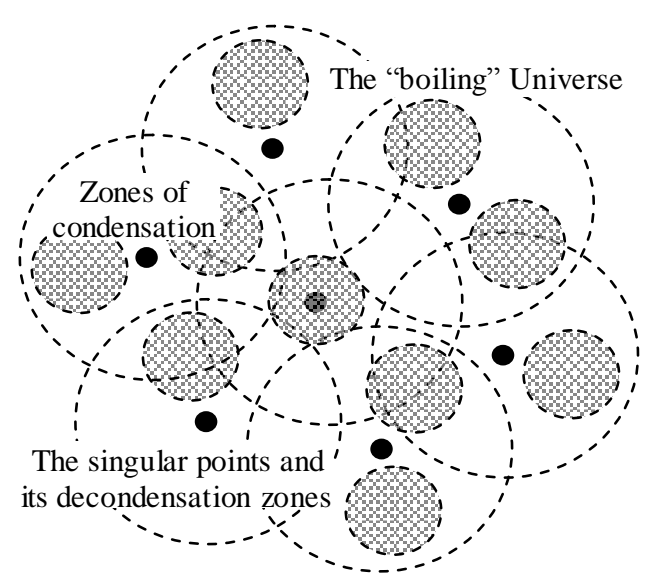

Figure 7. The scheme of the "boiling" Universe. Singular points with different UAP form different Universes. Singular points with the same UAP form zones of a unified Universe that is infinite because there are no other reasons. The set of singular points is infinite because they follow from the decaying Harmon. The STM-Complexes of singularity points are 
divided into separate Complexes, which lose strong mutual connectivity, become heterogeneous and form overlapping Real Worlds.

\section{The Hypothesis of the Inverted Universe's Cosmogony}

The Universe's cosmogony is deduced from the assumption of 1) infinite resources of Absolute Chaos and 2) finite resources of the produced from it entity per unit of Time produced from this Chaos. However, the revealed mechanism of such production in the absence of Time out of the Universe has an infinite carrier capacity and in the absence of restrictions on the quantity of the entity produced must instantly process the whole of the infinite Absolute Chaos and create an insoluble contradiction and an internal conflict in the external environment of the Universe.

In case of infinite resources of Absolute Chaos, it will create the Universe of the infinite degrees of infinity which is difficult to explain. And in case of finite resources of Absolute Chaos, it will instantly nullify these resources at admissible degrees of infinity of the Universe which is more plausible than the first assumption.

The obtained concept of the Universe's cosmogony is fair in both cases, but the first one (processing of Chaos) requires introduction of unknown restrictions on the carrier capacity of decondensation of Absolute Chaos, and the second one (processing of Harmony) is simpler, but assumes a zero initial resource of Absolute Chaos.

The inverted Universe's cosmogony uses the second method and assumes a zero initial resource of Absolute Chaos. The resource is continuously replenished with condensation of the completed zones of the Universe. The condensation instantly goes for decondensation into the Universe's entity and is returned back by the Universe into Absolute Chaos with continuous circulation according to this scheme.

In this case the Universe becomes an inverted Perpetuum Mobile of Absolute Chaos that is commonly known as a resource of any entity and its harmonization. In particular, such a mechanism serves as a refuse collector of the Universe's entities.

\section{The Universal Theory in Cosmogony Research}

A distinctive feature of universalization is mobilization of all known, especially the highest, Universe's concepts for the study of an object of research, the role which is played by the Universe in this paper. The methods of the universal research are applied as per many obligatory rather rigorous universal principles, the main of which is elimination of all external and internal contradictions and creation of quasi-universums of knowledge which is often absent in particular axiomatic research and, in particular, in the known cosmological models.

The obtained in such a way results are hypothetical as well as any other research, but the extent of their substantiation is usually larger than those of particular divided research, which are often included into the universal ones as particular cases, but they are given the other more adequate interpretations.

Therefore, despite the inevitable multivariance, every statement and substantiation made in this paper are deeply conceptually interrelated and stabilized among themselves, with the available facts, and research of the other authors as far as it is possible to do under modern conditions.

It is especially important for challenging research of extremely complex phenomena with limited conceptual and factual basis that is present in this theme.

The Universe's cosmogony is indirectly confirmed by an unconscious Mandala / Mangala symbol worship by nearly half of humanity, which is similar to the description of the Harmon as a producer of the whole entity, which is the evidence of the extinct civilizations which possessed the technologies of its use.

\section{Conclusion}

In this research complex universalization of the Universe cosmogony is investigated, covering all necessary stages of existence of the Universe and resolving the main external and internal contradictions unlike particular axiomatic theories. The structural formalism of cosmogony is obtained, developing generally accepted theories by means of fundamentally important concepts of Absolute Chaos, Harmon, Universe's Perpetuum Mobile, multivariance of the Universe, hypotheses of the "boiling" Universe, inverted Universe's cosmogony, etc. A great number of degrees of infinity and the possibility of unlimited multivariance of the Universe as well as the other interpretation of the known scientific data are substantiated.

This theory remains hypothetical, but the degree of its reliability must increase in the process of confirmation by new 
data and theories, and in any case, any consistent formalism must have its place in the infinite Universe's Chaos.

The Universe's cosmogony has great general scientific importance and can be used in different fields of knowledge due to the principled division of the whole entity into Chaos and Harmony. For example, universalization of intelligence (microcosm) revealed formal inheritance of the highest properties of the Universe (macrocosm) by all the living creatures to a different extent, and it underlies the emergence of life as a universal converter of Chaos into Harmony, irrespective of material realization, which has considerable importance in the Humanities, biology and creating thinking machines.

\section{References}

[1] A. Sosnitsky. Beginnings of the Universe Model and Deduction of Initial System of Information Concepts. Infor mation Theories \& Applications, 19 (1), 56-85, 2012.

[2] A. Sosnitsky. The Universal Meta Concept of Chaos. Proceedings of 8th CHAOS 2015 International Conference, 831-842, 2015.

[3] A. Einstein. Grundgedanken der allgemeinen Relativitätstheorie und Anwendung dieser Theorie in der Astronomie. Preussische Akademie der Wissenschaften, Sitzungsberichte, (Teil 1), 315, 1915.

[4] A. Friedman. Über die Krümmung des Raumes. Zeitschrift für Physik, 10 (1), 377-386, 1922.

[5] E. Hubble. A relation between distance and radial velocity among extra-galactic nebulae. PNAS, 15 (3), 168-173, 1929.

[6] S. Hawking, R. Penrose. The Singularities of Gravitational Collapse and Cosmology. Proc R. Soc. A, 314 (1519):

529-548, 1970.

[7] A. Guth. The Inflationary Universe: The Quest for a New Theory of Cosmic Origins. Perseus, 1997.

[8] String theory. Wikipedia, https://en.wikipedia.org/wiki/String_theory\#Early_results.

[9] J. Earman, J. Mosterín. A Critical Look at Inflationary Cosmology. Philosophy of Science, 66, 1-49, 1999.

[10] A. Sosnitsky. Harmonious Foundations of Intelligence. Communications of SIWN, 7, 66-72, 2009.

[11] A. She vchenko. The God. Nauka i Osvita, Kiev, 2017. 